\title{
Activity of Ceftaroline against Aerobic Gram-Positive and Gram-Negative Pathogens: Effect of Test Method Variability
}

\author{
Diane M. Citron, ${ }^{1}$ Yumi A. Warren, ${ }^{1}$ Kerin L. Tyrrell, ${ }^{1}$ and Ellie J. C. Goldstein ${ }^{1,2}$ \\ ${ }^{1}$ R.M. Alden Research Laboratory, Culver City, CA 90230, USA \\ ${ }^{2}$ Department of Medicine, David Geffen School of Medicine, Los Angeles, CA 90095, USA
}

Correspondence should be addressed to Ellie J. C. Goldstein, ejcgmd@aol.com

Received 23 August 2011; Accepted 28 September 2011

Academic Editor: P. D. Ghiringhelli

Copyright (C) 2011 Diane M. Citron et al. This is an open access article distributed under the Creative Commons Attribution License, which permits unrestricted use, distribution, and reproduction in any medium, provided the original work is properly cited.

\begin{abstract}
Ceftaroline is a new cephalosporin with bactericidal activity against methicillin-resistant S. aureus (MRSA) as well as gram-negative pathogens. Variations of in vitro test conditions were found to affect ceftaroline activity, with $5 \% \mathrm{NaCl}$ inhibiting growth and/or reducing the minimum inhibitory concentrations (MICs) for E. coli, K. pneumoniae, M. catarrhalis, H. influenzae, and streptococci, while an inoculum of $10^{6} \mathrm{CFU} / \mathrm{mL}$ raised MICs of some E. coli, K. pneumoniae, and M. catarrhalis strains.
\end{abstract}

\section{Introduction}

The emergence of MRSA has spurred the development of alternative therapies such as daptomycin, linezolid, and quinupristin-dalfopristin, which are not active against gram-negative pathogens and require combination therapy. Ceftaroline is a new, parenteral, broad-spectrum cephalosporin with bactericidal activity against MRSA, including vancomycinintermediate (VISA) strains, and multidrug-resistant Streptococcus pneumoniae (MDRSP); it is also active against common gram-negative pathogens and can therefore be used as monotherapy for mixed infections [1-6]. Since alterations of in vitro test conditions can potentially affect susceptibility results, we evaluated the effects of 15 variations to the standard test conditions as specified by the Clinical and Laboratory Standards Institute (CLSI) guidelines $[7,8]$ on the minimum inhibitory concentrations (MICs) of ceftaroline against 30 isolates representing 10 species of clinically important, commonly encountered organisms.

\section{Materials and Methods}

2.1. Standard Method. The CLSI reference broth microdilution method (CLSI 2006, 2009) uses cation-adjusted Mueller Hinton broth (CAMHB) (Difco, BD; Sparks, Md, USA), which has a calcium concentration of $25 \mathrm{mg} / \mathrm{L}$, a magnesium concentration of $12.5 \mathrm{mg} / \mathrm{L}$, and a pH of $7.3 \pm 0.1$. The standard inoculum is $5 \times 10^{5}$ colony-forming units $(\mathrm{CFUs}) / \mathrm{mL}$ for broth microdilution testing and $10^{4} \mathrm{CFU} /$ spot for agar dilution tests.

2.2. Test Variables. Modifications of standard test conditions included adjusting the $\mathrm{Ca}^{++}$content of CAMHB to $50 \mathrm{mg} / \mathrm{L}$ $\mathrm{Ca}$, addition of $\mathrm{NaCl}$ to $5 \%$, adjusting the broth to $\mathrm{pH} 6$ and $\mathrm{pH} 8$, and using inocula of $10^{4}$ and $10^{6}$ colony-forming units $(\mathrm{CFUs}) / \mathrm{mL}$. Other variations to the standard medium were the addition of $10 \%$ and $50 \%$ pooled human serum (Sigma; St. Louis, Mo, USA), the addition of lysed horse blood to 2.5\% (LHB) (Hardy Diagnostics, Inc. Santa Maria, Calif, USA), and using Haemophilus test medium (HTM) broth. While MIC panels were incubated at $35^{\circ} \mathrm{C}$ in ambient conditions, for comparative purposes, additional tests in CAMHB were incubated in the anaerobic chamber or in $5 \% \mathrm{CO}_{2}$.

2.3. MIC Test Panel Preparation. Ninety-six-well panels were prepared with twice the final concentration of ceftaroline (50 $\mu \mathrm{L} /$ well) using the Quick-Spense IIe apparatus (Sandy Springs Instruments; Germantown, Md, USA) and stored at $-70^{\circ} \mathrm{C}$ until used. Addition of $50 \mu \mathrm{L}$ of the organism inocula to the wells reduced the final ceftaroline concentration to 
TABLE 1: List of organisms used in the study.

\begin{tabular}{|c|c|c|c|c|}
\hline Organism & RMA number & Specimen source & Date isolated & Comments \\
\hline \multirow{3}{*}{ E. coli } & & ATCC 25922 & & \\
\hline & 19089 & Blood & $3 / 7 / 2007$ & Ampicillin $=4 \mu \mathrm{g} / \mathrm{mL}$ \\
\hline & 19090 & Primary infection site & $3 / 28 / 2007$ & Ampicillin $\geq 32 \mu \mathrm{g} / \mathrm{mL}$ \\
\hline \multirow{3}{*}{ K. pneumoniae } & 19091 & Primary infection site & $3 / 1 / 2007$ & Ampicillin $=32 \mu \mathrm{g} / \mathrm{mL}$ \\
\hline & 19092 & Blood & $6 / 6 / 2007$ & Ampicillin $=16 \mu \mathrm{g} / \mathrm{mL}$ \\
\hline & 19093 & Blood & $6 / 29 / 2007$ & Ampicillin $=32 \mu \mathrm{g} / \mathrm{mL}$ \\
\hline \multirow{3}{*}{ H. influenzae } & & ATCC 49247 & & \\
\hline & 16081 & Respiratory & $12 / 31 / 2003$ & $\beta$-Lactamase-negative \\
\hline & 18520 & Respiratory-sinus & $12 / 23 / 2005$ & $\beta$-Lactamase-positive \\
\hline \multirow{3}{*}{ M. catarrhalis } & 11940 & Respiratory-sinus & $6 / 14 / 2000$ & \\
\hline & 14032 & Respiratory-sinus & $5 / 22 / 2002$ & \\
\hline & 18861 & Respiratory-sputum & $1 / 31 / 2007$ & \\
\hline \multirow{6}{*}{ S. aureus } & & ATCC 29213 & & \\
\hline & 18488 & Chest infection site & $2 / 11 / 2005$ & Methicillin-S \\
\hline & 18401 & Blood & $8 / 16 / 2005$ & Methicillin-S \\
\hline & 18483 & Head abscess & $10 / 15 / 2005$ & Methicillin-R \\
\hline & 18504 & Primary infection site & $11 / 18 / 2005$ & Methicillin-R \\
\hline & 18526 & Blood & $10 / 24 / 2005$ & Methicillin-R \\
\hline \multirow{3}{*}{ E. faecalis } & & ATCC 29212 & & \\
\hline & 18284 & Foot infection site & $3 / 24 / 2005$ & \\
\hline & 18877 & Blood & $4 / 10 / 2007$ & \\
\hline \multirow{3}{*}{ S. pyogenes } & 17018 & Diabetic foot infection site & $1 / 22 / 2003$ & \\
\hline & 17019 & Diabetic foot infection site & $10 / 22 / 2002$ & \\
\hline & 19047 & Abdominal lesion & $10 / 26 / 2007$ & Clindamycin-R \\
\hline \multirow{6}{*}{ S. pneumoniae } & & ATCC 49619 & & \\
\hline & 19094 & Ear & $10 / 29 / 2007$ & Penicillin-S \\
\hline & 19095 & Eye & $1 / 9 / 2007$ & Penicillin-S \\
\hline & 13345 & Nasopharynx & $11 / 14 / 2001$ & Penicillin $=8 \mu \mathrm{g} / \mathrm{mL}$ \\
\hline & 13385 & Nasopharynx & $12 / 4 / 2001$ & Penicillin $=8 \mu \mathrm{g} / \mathrm{mL}$ \\
\hline & 18876 & Eye & $1 / 2 / 2007$ & Penicillin $=8 \mu \mathrm{g} / \mathrm{mL}$ \\
\hline
\end{tabular}

RMA: R.M. Alden (culture collection).

ATCC: American Type Culture Collection.

the desired level of 0.008 to $8 \mu \mathrm{g} / \mathrm{mL}$. Some of the organisms did not achieve a ceftaroline MIC endpoint, and further dilutions were prepared to $0.001 \mu \mathrm{g} / \mathrm{mL}$ for retesting some of those isolates.

2.4. Agar Dilution Test Media. Agar dilution MICs were determined on unsupplemented Mueller Hinton agar (MHA) (Difco), with 5\% LHB, and on HTM with $1.5 \%$ agar (HTMA). Serial twofold dilutions of ceftaroline were added to molten agar deeps to prepare the plates for use on the same day. Concentrations of ceftaroline ranged from 0.008 to $8 \mu \mathrm{g} / \mathrm{mL}$. Drug-free growth control plates were included (CLSI, 2006).

2.5. Test Organisms. All 30 strains tested were recent clinical isolates and American Type Culture Collection (ATCC) quality control (QC) strains, which included Escherichia coli ATCC 25922, Staphylococcus aureus ATCC 29213, Enterococcus faecalis ATCC 29212, Streptococcus pneumoniae ATCC 49619, and Haemophilus influenzae
ATCC 49247. Details about the clinical isolates are listed in Table 1. Clinical isolates were selected based on previously demonstrated resistance patterns. The isolates were stored in $20 \%$ skim milk at $-70^{\circ} \mathrm{C}$ and were taken from frozen stock and transferred twice on blood or chocolate agar (Hardy Diagnostics Inc.) before testing.

2.6. Inoculum Preparation for Microbroth Dilution Tests. Standard inocula were prepared by suspending colonies from overnight cultures in $0.85 \%$ saline to equal the turbidity of the $0.5 \mathrm{McF}$ arland standard and diluting it in CAMHB with the various additives at twice their final concentration, which upon addition of $50 \mu \mathrm{L}$ of inoculum to the test panel were diluted $1: 2$. The $10^{4}$ and $10^{6} \mathrm{cfu} / \mathrm{mL}$ inocula were prepared by diluting the saline suspension either 10 -fold more (for $10^{4} \mathrm{cfu} / \mathrm{mL}$ ) or 10 -fold less (for $10^{6} \mathrm{cfu} / \mathrm{mL}$ ). The trays were inoculated with $50 \mu \mathrm{L}$ of cell suspension for a final inoculum of $\sim 5 \times 10^{5} \mathrm{CFU} / \mathrm{mL}$ which was validated by 


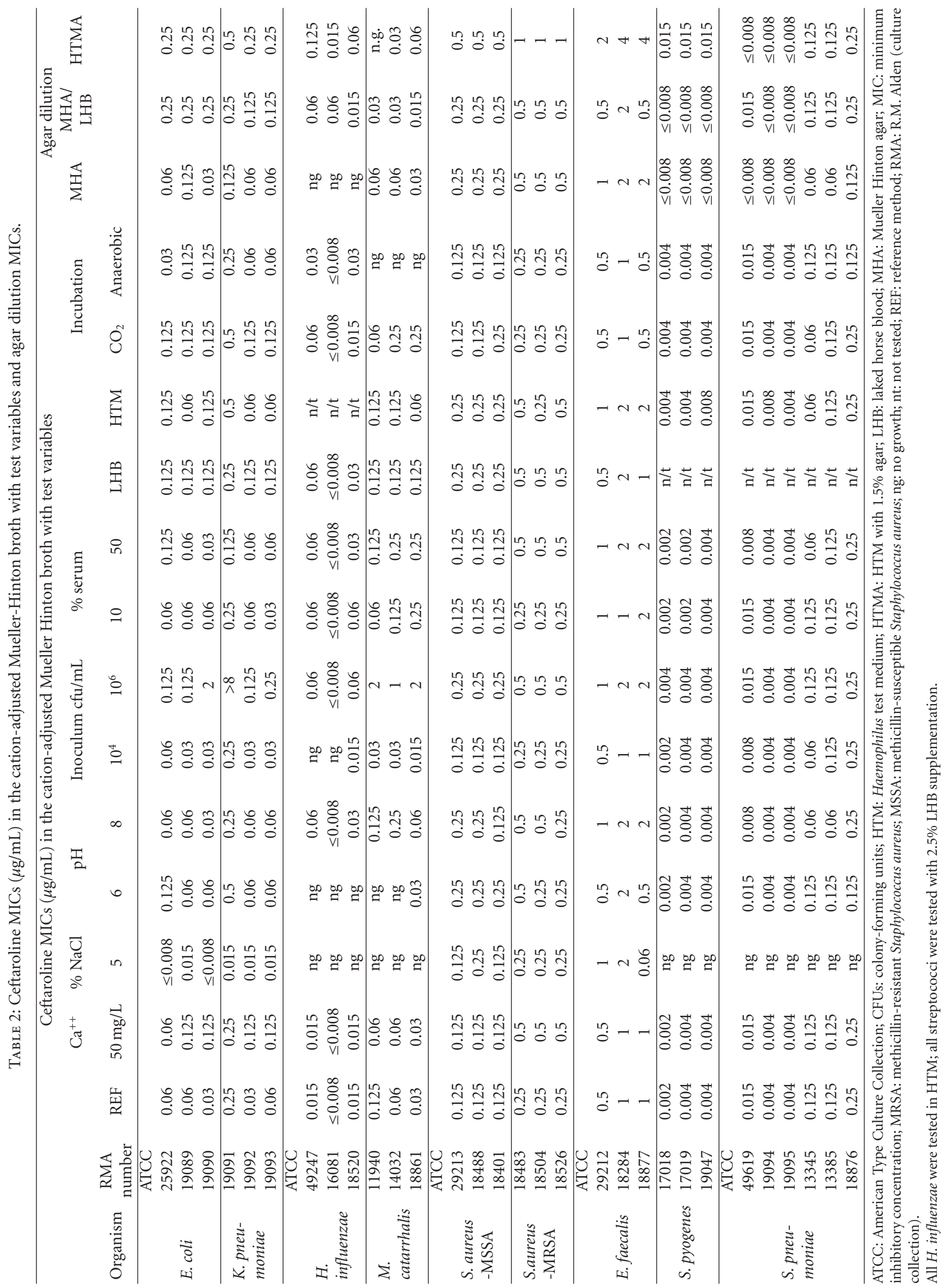


quantitative subculture from the growth control well. Inoculum preparation and all testing were performed in duplicate.

2.7. Agar Dilution Testing. For agar dilution tests, the cell suspensions prepared as above were diluted $1: 10$ in CAMHB and applied to the agar plates using a Steers replicator device that delivered a final inoculum of $10^{4} \mathrm{CFU} /$ spot.

2.8. MIC Determinations. After overnight incubation, the broth microdilution trays were examined for growth. The MIC was the lowest drug concentration that completely inhibited growth [7]. For agar dilution, the plates were incubated at $35^{\circ} \mathrm{C}$ overnight. The MIC was the lowest concentration that completely inhibited growth or resulted in a marked reduction of growth as compared with the drug-free control [7].

\section{Results}

We obtained MICs from duplicate tests under the variations shown in Table 2. In cases of discrepancy, the higher value was recorded. The ceftaroline MICs for the QC isolates (tested with the reference microbroth methods according to CLSI guidelines) were all within their acceptable ranges. Effects of variables in testing were noted where $5 \% \mathrm{NaCl}$ inhibited growth and/or reduced MICs for E. coli and K. pneumoniae and completely inhibited the growth of $M$. catarrhalis, $H$. influenzae, and all streptococci. Using an increased inoculum of $10^{6} \mathrm{cfu} / \mathrm{mL}$ increased the MIC 5 -fold for 1 of 3 E. coli strains that was also resistant to ampicillin (MIC $>32 \mu \mathrm{g} / \mathrm{mL}$ ) and 1 of $3 \mathrm{~K}$. pneumoniae strains that did not appear to have any unusual resistance pattern (ampicillin MIC $32 \mu \mathrm{g} / \mathrm{mL}$, ceftriaxone $0.25 \mu \mathrm{g} / \mathrm{mL}$ ). This $K$. pneumoniae isolate produced the same result when retested. The higher inoculum also increased MICs 3- to 5-fold for M. catarrhalis. The addition of blood or serum to the medium enhanced $M$. $c a$ tarrhalis growth without changing the MICs. Testing on agar, especially HTMA, produced MICs that were 1-3 dilutions higher. All other variables showed minimal effect, and the MICs were generally within one dilution of the reference method.

\section{Discussion}

Standardization of test conditions is an important factor in reliably reporting MICs. Yet variations of conditions may occur, and diverse adjustments may be employed by researchers for unusual testing circumstances worldwide. Jones et al. [9] studied the effects of modifying five parameters (11 variations) of in vitro testing of ceftaroline against 15 selected isolates. They found inocula of $5 \times 10^{7}$ raised the MICs from 8 to $>32$ and 0.5 to $>32 \mu \mathrm{g} / \mathrm{mL}$ on P. aeruginosa and E. cloacae, respectively. They also noted that $\mathrm{pH} 5.0$ impaired the growth of 9/12 organisms and consequently lowered their MICs and that increased calcium concentration lowered the MICs of E. faecalis and E. faecium, but that the addition of serum or LHB and variable incubation conditions did not affect ceftaroline MICs. Our study differed from theirs by including a larger number of isolates and adding such variables as $5 \%$ $\mathrm{NaCl}$, serum at 10 and $50 \%$ concentrations, variations in incubation conditions, and while there was some overlap in species, they did not report on the respiratory pathogens $H$. influenza and M. catarrhalis, nor did they study S. pyogenes or K. pneumoniae. While some of our test variations were in accord with those of Jones et al., they differed in several respects as well. Jones et al. [9] noted that high inocula did not affect E. coli MICs but did raise E. cloacae and P. aeruginosa MICs, but we found a fourfold increased MIC for one of three E. coli strains tested as well as a fivefold increase with one of three K. pneumonia isolates, suggesting strain variation. Additionally, we found that $M$. catarrhalis, $H$. influenza, and $\mathrm{S}$. pyogenes grew poorly with $\mathrm{NaCl}$ supplementation and at $\mathrm{pH}$ 6.0. An inoculum of $10^{6} \mathrm{CFU} / \mathrm{mL}$ also increased the MICs four- to six-fold for all three M. catarrhalis strains tested.

\section{Conclusion}

The in vitro antibacterial activity of ceftaroline was adversely affected by $5 \% \mathrm{NaCl}$ which inhibited growth and/or reduced MICs for E. coli, K. pneumoniae, M. catarrhalis, H. influen$z a e$, and streptococci, while an inoculum of $10^{6} \mathrm{CFU} / \mathrm{mL}$ raised MICs of some E. coli, K. pneumoniae, and $M$. catarrhalis strains. The other modifications tested did not adversely affect MIC results. Organisms with special growth requirements can be tested for ceftaroline susceptibility with reasonable assurance that test conditions will not affect the MIC results.

\section{Acknowledgment}

This study was supported by a grant from the Forest Laboratories, Inc.

\section{References}

[1] C. Jacqueline, J. Caillon, V. Le Mabecque et al., "In vivo efficacy of ceftaroline (PPI-0903), a new broad-spectrum cephalosporin, compared with linezolid and vancomycin against methicillin-resistant and vancomycin-intermediate Staphylococcus aureus in a rabbit endocarditis model," Antimicrobial Agents and Chemotherapy, vol. 51, no. 9, pp. 3397-3400, 2007.

[2] S. Mushtaq, M. Warner, Y. Ge, K. Kaniga, and D. M. Livermore, "In vitro activity of ceftaroline (PPI-0903M, T-91825) against bacteria with defined resistance mechanisms and phenotypes," Journal of Antimicrobial Chemotherapy, vol. 60, no. 2, pp. 300311, 2007.

[3] D. Parish and N. Scheinfeld, "Ceftaroline fosamil, a cephalosporin derivative for the potential treatment of MRSA infection," Current Opinion in Investigational Drugs, vol. 9, no. 2, pp. 201209, 2008

[4] H. S. Sader, T. R. Fritsche, K. Kaniga, Y. Ge, and R. N. Jones, "Antimicrobial activity and spectrum of PPI-0903m (T-91825), a novel cephalosporin, tested against a worldwide collection of clinical strains," Antimicrobial Agents and Chemotherapy, vol. 49, no. 8, pp. 3501-3512, 2005.

[5] H. S. Sader, T. R. Fritsche, and R. N. Jones, "Antimicrobial activities of ceftaroline and ME1036 tested against clinical 
strains of community-acquired methicillin-resistant Staphylococcus aureus," Antimicrobial Agents and Chemotherapy, vol. 52, no. 3, pp. 1153-1155, 2008.

[6] G. H. Talbot, D. Thye, A. Das, and Y. Ge, "Phase 2 study of ceftaroline versus standard therapy in treatment of complicated skin and skin structure infections," Antimicrobial Agents and Chemotherapy, vol. 51, no. 10, pp. 3612-3616, 2007.

[7] Clinical and Laboratory Standards Institute, "Methods for dilution antimicrobial susceptibility tests for bacteria that grow aerobically; approved standard-sixth edition," Clinical and Laboratory Standards Institute Document M7-A6, Clinical and Laboratory Standards Institute, Wayne, Pa, USA, 2006.

[8] Clinical and Laboratory Standards Institute, "Performance standards for antimicrobial susceptibility testing; 19th informational supplement," document M100-S19, Clinical and Laboratory Standards Institute, Wayne, Pa, USA, 2009.

[9] R. N. Jones, T. R. Fritsche, Y. Ge, K. Kaniga, and H. S. Sader, "Evaluation of PPI-0903M (T91825), a novel cephalosporin: bactericidal activity, effects of modifying in vitro testing parameters and optimization of disc diffusion tests," Journal of Antimicrobial Chemotherapy, vol. 56, no. 6, pp. 1047-1052, 2005. 

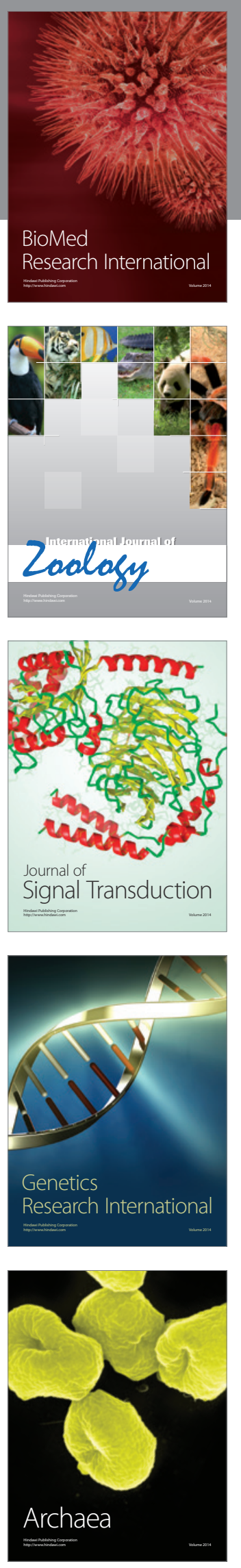
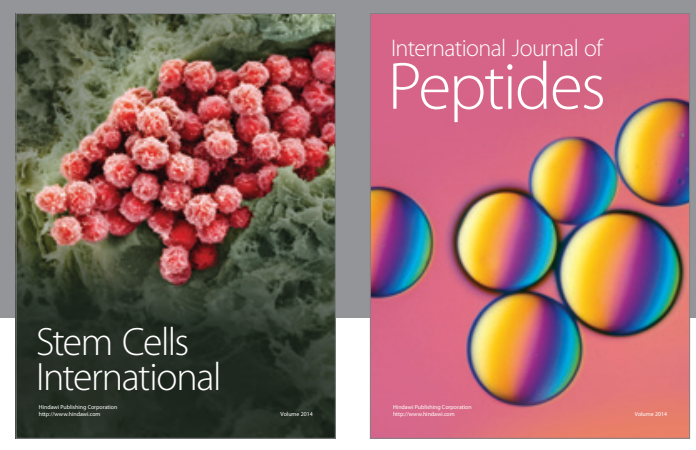

Submit your manuscripts at

http://www.hindawi.com
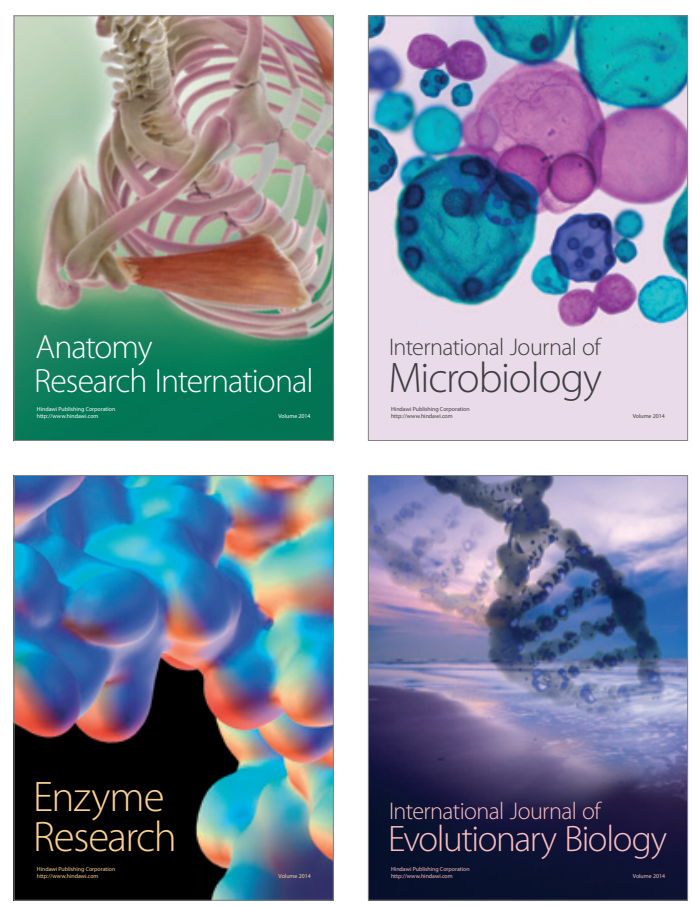
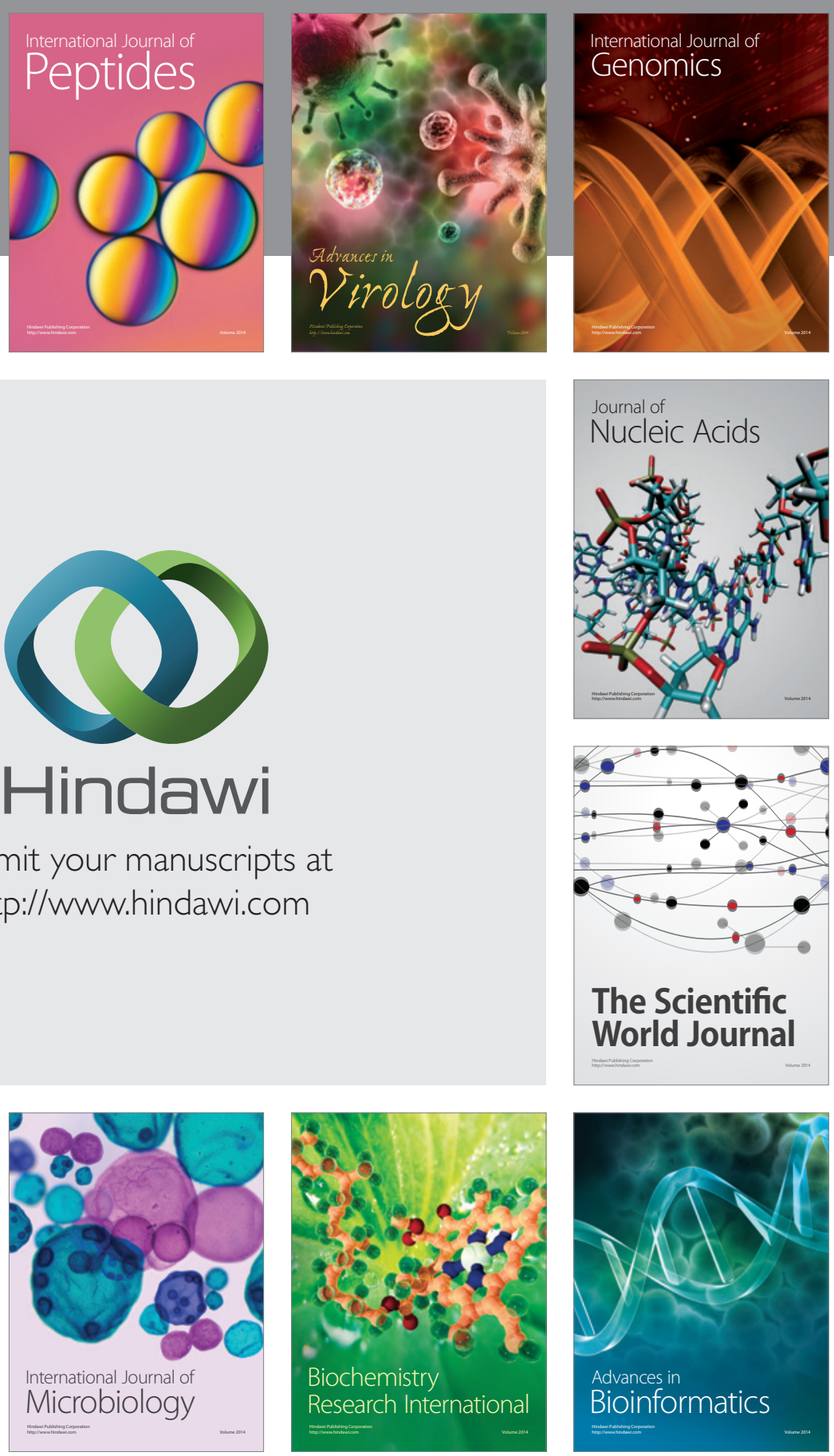

The Scientific World Journal
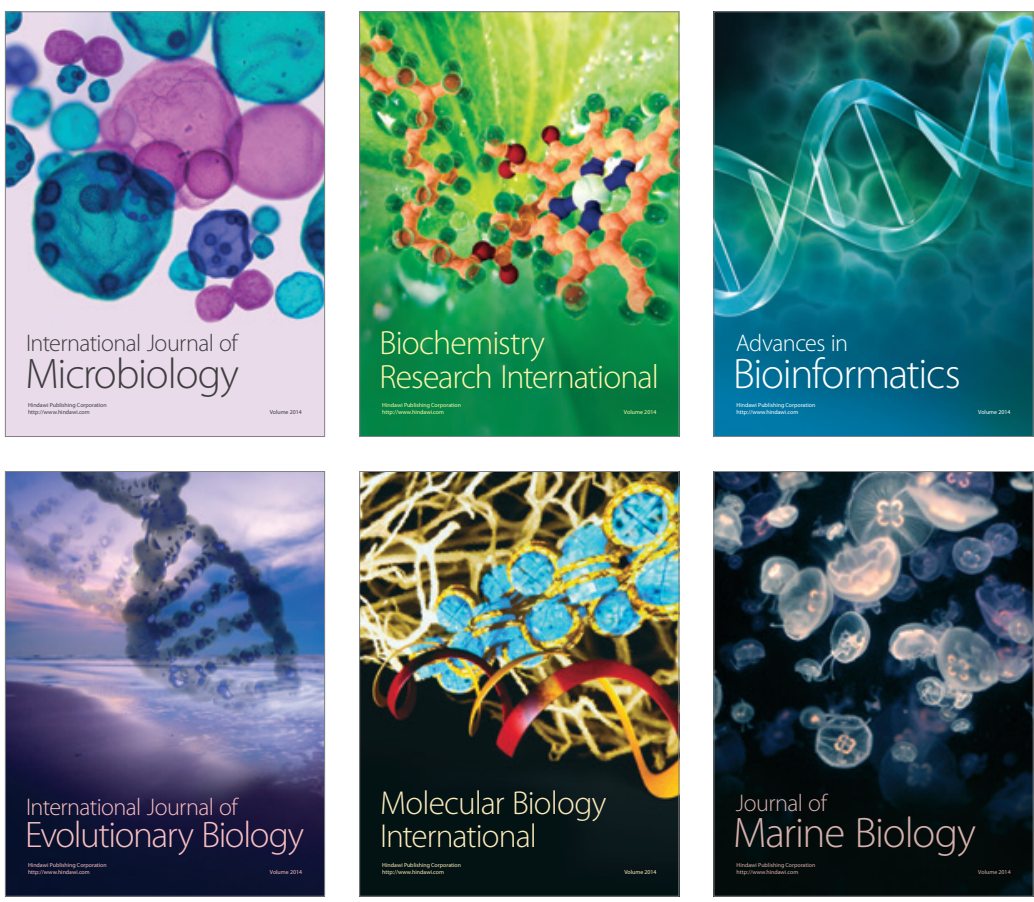